\title{
SPATIALLY-VARYING IIR FILTER BANKS FOR IMAGE CODING
}

\author{
Wilson C. Chung and Mark J. T. Smith \\ School of Electrical Engineering \\ Georgia Institute of Technology \\ Atlanta, GA 30332
}

\begin{abstract}
This paper reports on the application of spatially variant IIR filter banks to subband image coding. The new filter bank is based on computationally efficient recursive polyphase decompositions that dynamically change in response to the input signal. In the absence of quantization, reçonstruction can be made exact. However, by proper choice of an adaptation scheme, we show that subband image coding based on time varying filter banks can yield improvement over the use of conventional filter banks.
\end{abstract}

\section{INTRODUCTION}

Recursive analysis and synthesis filter banks for subband coding have been considered previously and were shown to be particularly attractive because they preserve exact reconstruction and can achieve tremendous computational efficiency relative to comparable FIR systems [6], [8]. These filter banks are typically two-band systems that serve as building blocks for uniform and non-uniform band treestructured systems.

Much attention has been given in minimizing the perceptually significant coding distortions in image coding systems. At low rates, aliasing and ringing distortions tend to be pronounced and have sperned some efforts to reduce these effects; but the success has been limited [9], [6]. The difficulty is that the step response ripples of the filters, which are the source of the ringing distortion, are needed to achieve good filter magnitude response characteristics. Good spectral magnitude characteristics are important for reducing the effects of aliasing in the presence of coding. Aliasing distortion appears as blurring in the image and is subjectjvely noticeable. If filters with good magnitude characteristics are used to reduce the aliasing, then ringing distortion results which is also very noticeable and objectionable. Since both good step response and good magnitude response characteristics can not be achieved simultaneously for conventional filter banks, this poses a dilemma.

A solution to this problem using IIR time-varying filter banks was proposed at the IEEE DSP Workshop [2] and a theoretical analysis of the filter bank was presented in [4]. In this paper, we expand upon this idea giving special attention to its application to image coding. In the next section, we briefly summarize the results derived in [4]

This work is supported in part by the National Science Foundation under contract MIP-9116113 and the National Aeronautics and Space Administration.

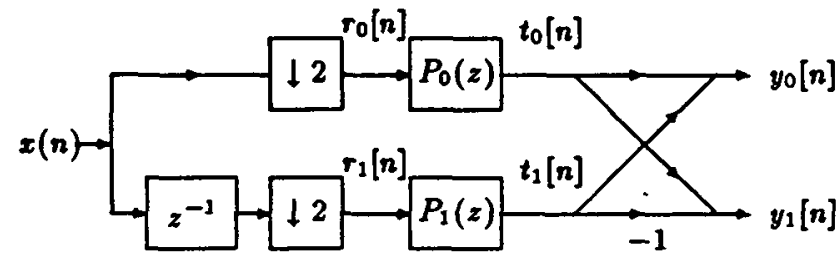

(a) Analysis

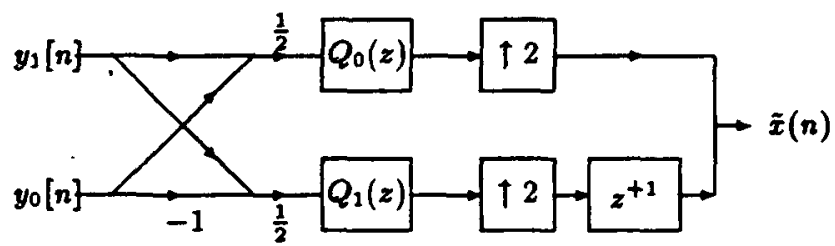

(b) Synthesis

Figure 1: Two-Band Polyphase Implementation of the Analysis/Synthesis System (a) Analysis, (b) Synthesis

which are the basis for this work. In the follow section we consider a control mechanism for performing the adaptation and compare the performance of the overall system to that of a conventional system.

\section{SPATIALLY-VARIANT FILTER BANKS}

The two-band polyphase system, as shown in Figure 1, is commonly used in conventional subband analysis/synthesis systems. The lowpass and highpass analysis filters, $H_{0}(z)$, and $H_{1}(z)$, have the form:

$$
\begin{aligned}
& H_{0}(z)=P_{0}\left(z^{2}\right)+z^{-1} P_{1}\left(z^{2}\right) \\
& H_{1}(z)=P_{0}\left(z^{2}\right)-z^{-1} P_{1}\left(z^{2}\right)
\end{aligned}
$$

where $P_{0}(z)$ and $P_{1}(z)$ are the analysis polyphase filters and $Q_{0}(z)$ and $Q_{1}(z)$ are the synthesis polyphase filters. This system has the property that its structure guarantees exact
(NASA-CR-193727) SPATIALLY-VARYING
IIR FILTER BANKS FOR IMAGE CODING
(Georgia InSt. of Tech.) $4 \mathrm{p}$
N94-12897 
reconstruction when

$$
Q_{0}(z)=\frac{1}{P_{0}(z)} \quad \text { and } \quad Q_{1}(z)=\frac{1}{P_{1}(z)} .
$$

The polyphase filters, $P_{0}(z)$ and $P_{1}(z)$, are assumed to be causal stable recursive filters with zeros strictly outside the unit circle and have allpass or near allpass characteristics. The synthesis polyphase filters, $Q_{0}(z)$ and $Q_{1}(z)$, by definition will have their poles outside the unit circle and their zeros inside the circle. Consequently they are anti-cansal and stable.

The new spatially-varying system is identical except that the filters change in response to the input. In particular, the coefficients of the polyphase filters $P_{0}(z)$ and $P_{1}(z)$ are changed selectively at some point in the filtering process to another set of polyphase filters. Thus to preserve exact reconstruction it is sufficient to guarantee that $r_{i}[n]=t_{i}[n]$ (where $i=0,1$ ) at all times before, during, and after the switching of coefficients. The derivation presented in [4] considered starting with a set of polyphase filters $\left(p_{0}^{2}[n]\right.$, $\left.p_{1}^{L}[n]\right)$ and switching them to another set of filters $\left(p_{0}^{k}[n]\right.$, $\left.p_{1}^{\mathcal{R}}[n]\right)$. For convenience, assume that we switch the filters at $n=0$ and that the polyphase filters have the form:

$$
p^{L}(z)=\frac{\alpha_{0}^{L}+\alpha_{1}^{L} z^{-1}+\cdots+\alpha_{M_{0}}^{L} z^{-M_{0}}}{1+\beta_{1}^{L} z^{-1}+\beta_{2}^{L} z^{-2}+\cdots+\beta_{N_{0}}^{L} z^{-N_{0}}} .
$$

and

$$
p^{R}(z)=\frac{\alpha_{0}^{R}+\alpha_{1}^{R} z^{-1}+\cdots+\alpha_{M_{1}}^{R} z^{-M_{1}}}{1+\beta_{1}^{R} z^{-1}+\beta_{2}^{R} z^{-2}+\cdots+\beta_{N_{1}}^{R} z^{-N_{2}}} .
$$

The labels $L$ and $R$ signify that the signal or filter coefficient is associated with the left and right half of the time index respectively.

The direct form difference equations that implement the polyphase filters are:

$$
v_{L}[n]=\sum_{m=0}^{N_{0}} \alpha_{m}^{L} x[n-m]-\sum_{\ell=1}^{N_{0}} \beta_{l}^{L} v_{L}[n-\ell]
$$

in the range $-\infty<n \leq-1$ and

$$
v_{R}[n]=\sum_{m=0}^{M_{1}} \alpha_{m}^{R} x[n-m]-\sum_{\ell=1}^{N_{1}} \beta_{\ell}^{R} v_{R}[n-\ell]
$$

when $0 \leq n<\infty$. Note that $v_{L}[n]$ and $v_{R}[n]$ are the leftsided and right-sided components of a polyphase filter output $v[n]$, i.e. $v[n]=v_{L}[n]+v_{R}[n]$. Equation (4) requires the initial conditions $v_{R}\left[-N_{1}\right], v_{R}\left[-N_{1}-1\right], \ldots, v_{R}[-1]$ in order to evaluate $v_{R}[0]$. These initial conditions are obtained from the past samples of the ontput, i.e. $v_{R}[n]=v_{L}[n]$ in the range $-N_{1} \leq n \leq-1$.

The reconstruction equations (which are derived in [4]) are performed by anti-causal filtering and are defined by the difference equations

$$
\begin{array}{r}
x_{R}\left[n-M_{1}\right]=\frac{1}{-\alpha_{M_{1}}^{R}} \sum_{\ell=1}^{M_{1}} \alpha_{M_{1}-\ell}^{R} x_{R}\left[n+\ell-M_{1}\right]+ \\
\frac{1}{\alpha_{M_{1}}^{R}} \sum_{m=0}^{N_{1}} \beta_{N_{1}-m}^{R} v_{R}\left[n+m-N_{1}\right]
\end{array}
$$

for $0 \leq n<\infty$ and

$$
\begin{array}{r}
x_{L}\left[n-M_{0}\right]=\frac{1}{-\alpha_{M_{0}}^{L}} \sum_{\ell=1}^{M_{0}} \alpha_{M_{0}-\ell}^{L} x_{L}\left[n+\ell-M_{0}\right]+ \\
\frac{1}{\alpha_{M_{0}}^{L}} \sum_{m=0}^{N_{0}} \beta_{N_{0}-m}^{L} v_{L}\left[n+m-N_{0}\right]
\end{array}
$$

for $-\infty<n \leq-1$. The assumption that the switching occurs at $n=0$ was done purely for convenience. But clearly these equations hold regardless of when the filters are switched. However, there is a constraint on how close together we can switch our filter. Switching points should occur at least $N_{0}$ samples apart. In the next section, we consider how to determine when to switch a filter and what characteristics the filters should have in order to improve subjective and objective performance.

\section{ADAPTATION CONTROL SYSTEM}

The flexibility to switch among a set of different filters is potentially very powerful. But the key to make this work well is determining an effective control system for adapting the filters relative to the signal. Obviously there are many possibilities. The one investigated in this work is based on the observation that filters with good step response characteristics tend to perform better in local regions with sharp edges or transitions. Filters with good magnitude response characteristics seem to be better suited for the other regions. Thus two sets of filters are used in this system: filter set $A$, which has good magnitude response characteristics; and filter set $B$, which has good step response characteristics. Figure 3 shows the spectral magnitude response for the lowpass filters in sets $A$ and B. Notice that lowpass filter $A$ has good attenuation and transition characteristics while lowpass filter $B$ does not. Figure 2 shows the step response for these filters. Here lowpass filter $B$ has an almost ripple free characteristic while lowpass filter $A$ does not.

The remaining question is how to switch between these filters. Several points within the structure (see Figure 1) can be used to estimate an optimal switch point. For example, $x[n], r_{0}[n], t_{0}[n]$, or $y_{0}[n]$ represent estimation points in the structure that could be used to estimate sharp signal transition regions. The estimation procedure used here consists of computing the first backward difference of the signal taken at one of the estimation points mentioned above. When the magnitude of the first backward difference exceeds a pre-set threshold, we consider this to be a sharp transition and switch to filter set $B$. When that transition period is over, we switch back to filter set $A$. It is important to the performance of the system to switch the filters prior to and not during the transition. Therefore once a transition point is detected by the first backward difference estimator, we move back a few samples to the onset of the transition. Similarly when the estimator falls below the a lower threshold, the filter is switched back to filter set $A$.

In the case where $x[n]$, $r_{0}[n]$, or $t_{0}[n]$ are used as estimation points, side information must be transmitted to indicate the location of the switch point. For natural images, the amount of side information is generally negligible. For example, for the test image Lena, only about 0.0281 
$A$

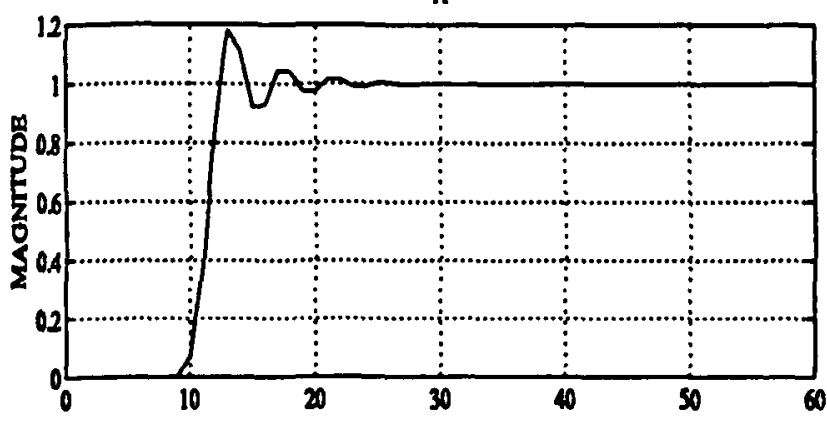

B

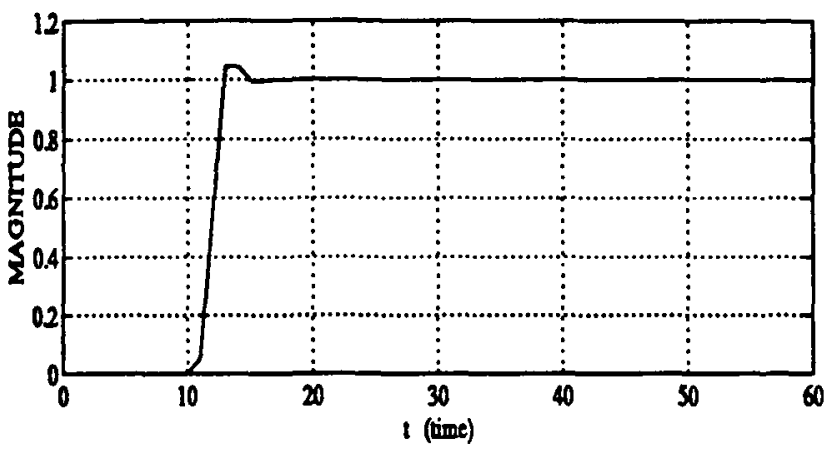

Figure 2: Step response characteristics for the lowpass filters $A$ and $B$.

bits/pixel was consumed by this side information. If, on the other hand, yo[n] is used as an estimation point, sending side information can be avoided. More precisely, the estimation should be performed on $y[n]$ after it has been coded. This allows the synthesis section to derive the switching point from the exact same signal used by the analysis section to derive the switching point. This form of the implementation is a feedback adaptation approach. Interestingly, there is often enough information in the $y_{0}[n]$ signal to make an accurate estimation of major transitions. Even if the estimation is in error occasionally, the results are not catastrophic since the system performance is lower bounded by that of conventional subband coding (which is not bad).

\section{SIMULATION RESULTS}

To evaluate the new analysis/synthesis system, we considered a baseline pyramid two-level system with seven subbands and the Enumerative Laplacian Quantization (ELQ) encoder introduced by Darragh [10]. Further, we used the method of circular convolution to periodically extend our image boundaries [6]. The derivation of initial conditions for 2-D exact reconstruction with IIR filters is addressed in [6]. The $256 \times 256$ test image Lena was used in this example. A section of the original is shown in Figure 4. Figure 5 shows Lena coded at 0.4527 bits/pixel with conventional IIR filter banks. The filters used are recursive (filter set A) with magnitude response and step response plots shown in Figures 3 and 2. The output corresponding to the new system is shown in Figure 6 . It is coded at the same bit

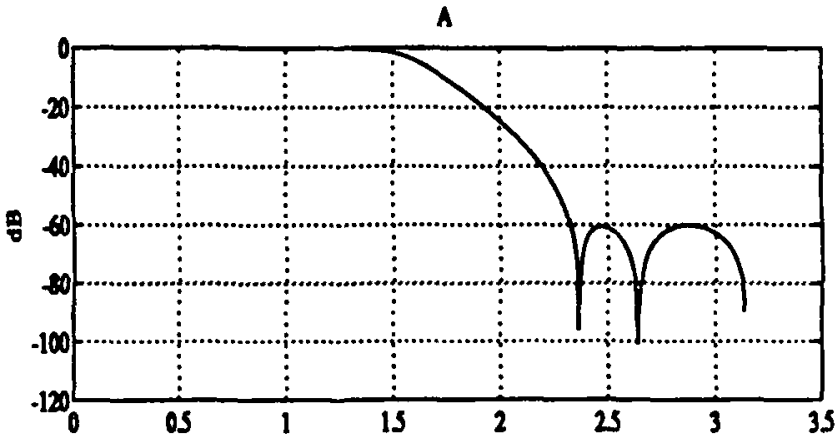

B

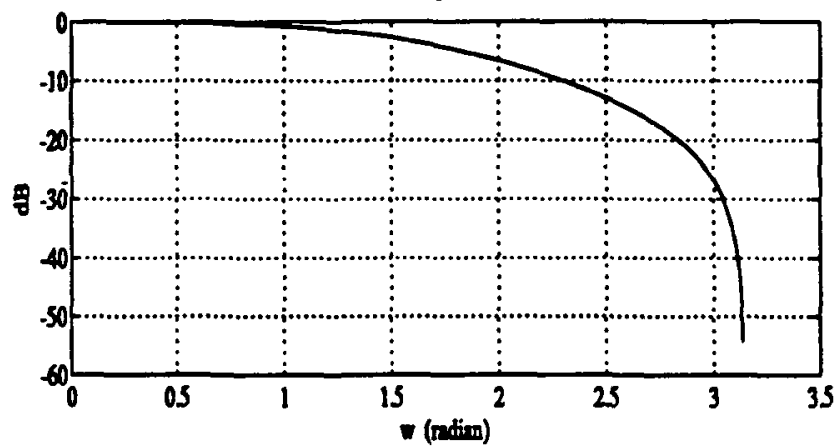

Figure 3: Magnitude response characteristics for the lowpass filters $A$ and $B$.

rate and uses $r_{0}[n]$ as an estimation point. Approximately $0.0281 \mathrm{bits} / \mathrm{pixel}$ are devoted to the side information associated with the adaptation. In our feedforward system, we use an arithmetic coder as a lossless coder for the side information. The subjective quality is noticably better as shown by examining both coded outputs. The ringing distortion is quite noticeable around the boundary of the hat, as seen in 5. On the other hand, the ringing distortion disappeared in the coded image using the new system, as seen in 6 . Little magnitude distortion can be seen around the edges since the filter set of good step response and bad magnitude response is allowed to switch back very quickly after only a few samples. This improvement in quality is also reflected in the PSNR of the coded results.

Several important aspects should be addressed in optimizing the performance of the new coder. First, the intelligent selection of threshold levels in determining the edges or boundaries within an image is critical. A good edge or level detector almost surely guarantees the success of the adaptation control system. Secondly, the issue of signal extension should be study carefully in light of the new spatially variant system because the derivation of initial conditions no longer depends on only one distinct filter set, but manf. Finally, a feedback scheme can be considered where side information for edge locations within an image will no longer be needed apriori for transmission over the channel. As with most image coding strategies, much of the improvement in quality comes as a result of fine tuning the coder. This has not be done yet at this point but should be done in time for presentation at the conference. 


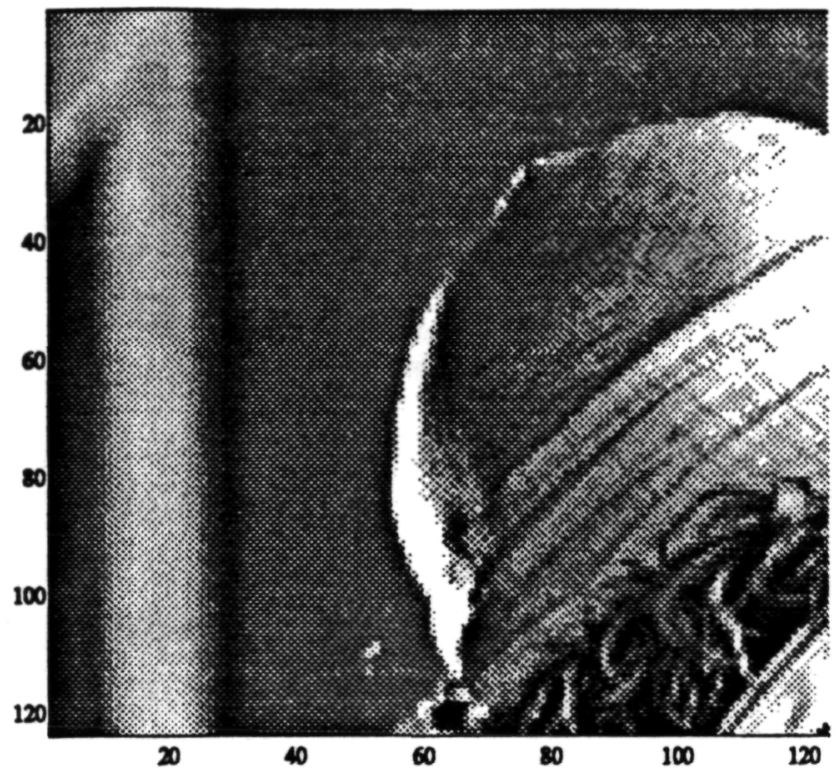

Figure 4: Original test image Lena

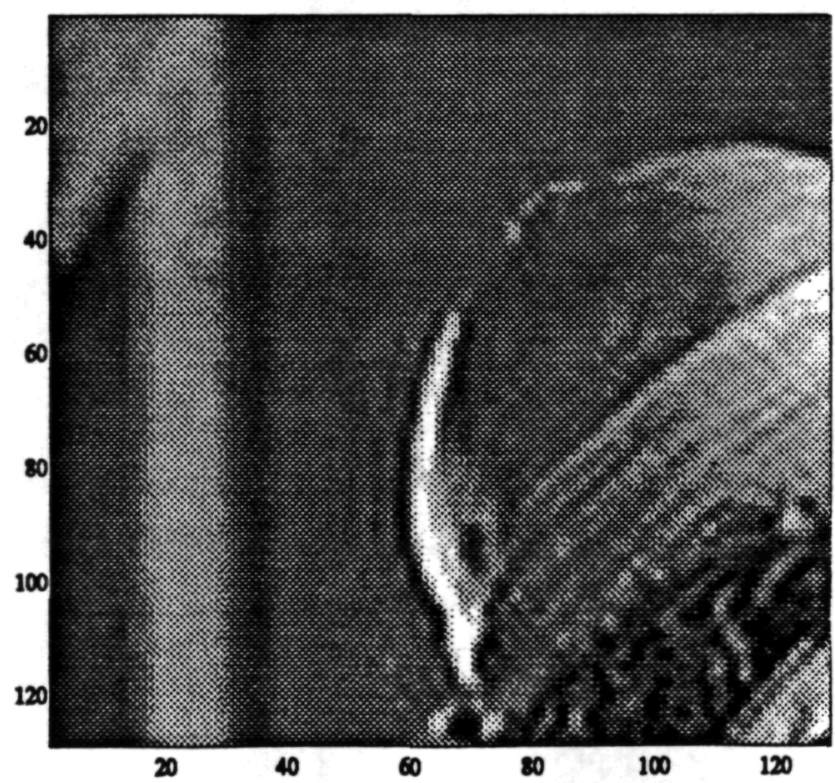

Figure 5: Lena coded at 0.4527 bits/pixel using the conventional IIR filter banks with filter $A$ as the basis filters. The ELQ coder is used to code the subband images. (PSNR = 28.91 dB)

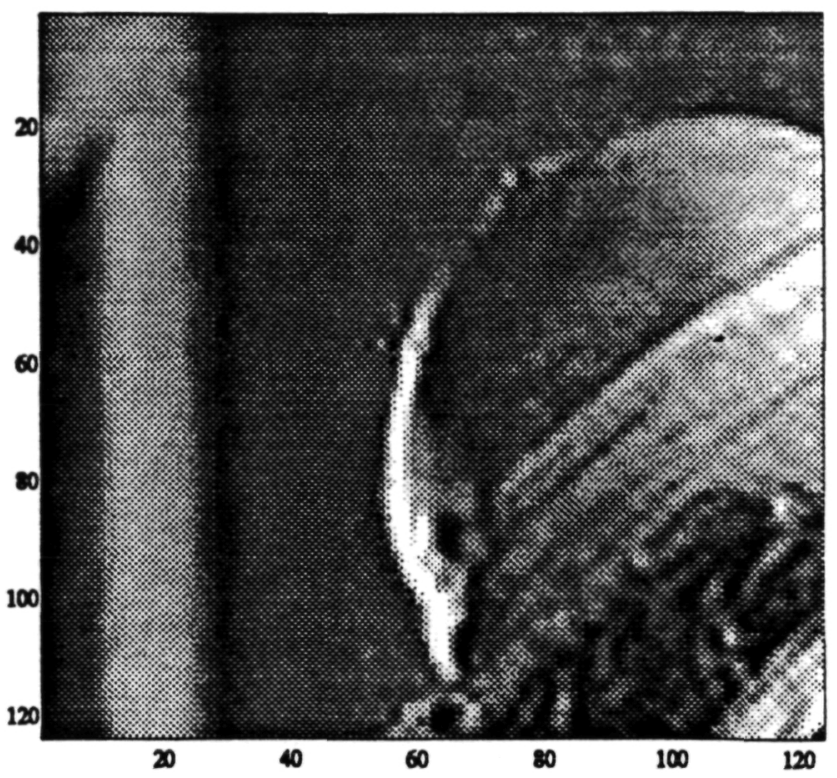

Figure 6: Lena coded at 0.4527 bits/pixel using the new spatially-varying IIR filter banks. Filters $A$ and $B$ are employing in the switching. The ELQ coder is used to code the subband images. (PSNR $=29.21 \mathrm{~dB}$ )

\section{REFERENCES}

[1] J. W. Woods and S. D. O'Neil, "Subband coding of images," IEEE Trans. Acoust., Speech, Signal Processing, ASSP-34, pp. 1278-1288, Oct. 1986.

[2] M. J. T. Smith and W. C. Chung, "Adaptive IIR Analysis-Synthesis Filter Banks," IEEE DSP Workshop, September 16-19, 1992, Chicago, Illinois.

[3] M. J. T. Smith and W. C. Chung, "Recursive TimeVarying Filter Banks for Subband Image Coding," Submitted to IEEE Trans. on Image Processing,

[4] M. J. T. Smith and W. C. Chung, "Spatially Adaptive IIR Analysis-Synthesis Filter Banks for Image Coding," Proceedings of the Asilomar Conference, October, 1992.

[5] Subband Image Coding, (John Woods, Ed.), Kluwer Academic Publishers, Copyright 1991.

[6] M. J. T. Smith and S. L. Eddins, “Analysis/synthesis techniques for subband image coding," IEEE Trans. on ASSP, August 1990.

[7] G. Karlsson and M. Vetterli, “Sub-band coding of finite length signals," Signal Processing, volume 17, pp. 161168, June 1989.

[8] J. H. Husoy, Subband Coding of Still Images and Video, Ph.D. Thesis, University of Trondheim, Norway, 1991.

[9] T. Kronander, Some Aspects of Perception Based Image Coding, $\mathrm{Ph}$.D. Thesis, Linkoping University, Sweden, 1989.

[10] J. Darragh, Subband and Transform Coding of Images, Ph.D. Thesis, UCLA, 1989. 